\title{
Soliton, kink and antikink solutions of a 2-component of the Degasperis-Procesi equation
}

\author{
Jiangbo Zhou*, Lixin Tian, Xinghua Fan \\ Nonlinear Scientific Research Center, Faculty of Science, Jiangsu University, Zhenjiang, \\ Jiangsu 212013, China
}

\begin{abstract}
In this paper, we employ the bifurcation theory of planar dynamical systems to investigate the traveling wave solutions of a 2-component of the Degasperis-Procesi equation. The expressions for smooth soliton, kink and antikink solutions are obtained.

Key words: 2-component of the Degasperis-Procesi equation, bifurcation method, soliton, kink solution, antikink solution

2000 MSC: 35Q51, 34C23, 37G10, 35Q35
\end{abstract}

\section{Introduction}

Since the theory of solitons has very wide applications in fluid dynamics, nonlinear optics, biochemistry, microbiology, physics and many other fields, the study of soliton solutions has become one of the important issues of nonlinear partial differential equations [1]-[11].

In 1999, Degasperis and Procesi [12] derived a nonlinear dispersive equa-

\footnotetext{
*Corresponding author

Email address: zhoujiangbo@yahoo.cn (Jiangbo Zhou)
} 
tion

$$
u_{t}-u_{x x t}+4 u u_{x}=3 u_{x} u_{x x}+u u_{x x x}
$$

which is called the Degasperis-Procesi equation. Here $u(t, x)$ represents the fluid velocity at time $t$ in the $x$ direction in appropriate nondimensional units (or, equivalently the height of the water's free surface above a flat bottom). The nonlinear convection term $u u_{x}$ in Eq.(1.1) causes the steepening of wave form, whereas the nonlinear dispersion effect term $3 u_{x} u_{x x}+u u_{x x x}=\left(\frac{1}{2} u^{2}\right)_{x x x}$ in Eq.(1.1) makes the wave form spread. Eq.(1.1) can be regarded as a model for nonlinear shallow water dynamics [13].

An important issue regarding the Degasperis-Procesi equation is to find its traveling wave solutions. Vakhnenko and Parkes [14] derived periodic and solitary wave solutions of Eq.(1.1). Matsuno [15]-[17] obtained multisoliton, $N$-soliton, cusp and loop soliton solutions of Eq.(1.1). Lundmark and Szmigielski [18] investigated multi-peakon solutions of Eq.(1.1). Shock wave solutions of Eq.(1.1) were obtained in [19]. Lenells [20] classified all its weak traveling wave solutions. Chen and Tang [21] showed that Eq.(1.1) has kink-like and antikink-like wave solutions. Qiao [8] obtained the peakons, dehisced solitons, cuspons and new 1-peak solitons of Eq.(1.1).

It is known that the Degasperis-Procesi equation has solitons, but has no kink or antikink solutions. In this paper, we generalize the Degasperis-Procesi equation to the following 2-component of Degasperis-Procesi equation

$$
\left\{\begin{array}{l}
m_{t}+u m_{x}+3 u_{x} m=v_{x} \\
v_{t}=(u v)_{x}
\end{array}\right.
$$

where $m=u-u_{x x}$. This 2-component of the Degasperis-Procesi equation can be applied to describe shallow water waves. In Eqs.(1.2), $u(x, t)$ denotes 
the height of the water surface above a horizontal bottom and $v(x, t)$ is related to the horizontal velocity field. Obviously, if $v \equiv 0$, then Eqs.(1.2) is reduced to the Degasperis-Procesi equation (1.1). Using the bifurcation theory of planar dynamical systems (see [22]), we will show that, in addition to smooth solitons, Eqs.(1.2) has kink and antikink solutions. The solitons denote the nonlinear localized waves on the shallow water's free surface that retain their individuality under interaction and eventually travel with their original shapes and speeds. The kink and antikink waves, which rise or descend from one asymptotic state to another, form another type. The balance between the nonlinear steepening and dispersion effect under Eqs.(1.2) gives rise to these solutions. The solutions presented in this paper may help people to know deeply the described physical process of Eqs.(1.2).

The remainder of the paper is organized as follows. In Section 2, using the traveling wave transformation, we transform Eqs.(1.2) into a planar dynamical system and then discuss bifurcations of phase portraits of this system. In Section 3, we obtain the expressions for the smooth soliton, kink and antikink solutions of Eqs.(1.2). A short conclusion is given in Section 4 .

\section{Bifurcation and phase portraits of the traveling wave system}

Eqs.(1.2) also takes the form

$$
\left\{\begin{array}{l}
u_{t}-u_{x x t}+4 u u_{x}=3 u_{x} u_{x x}+u u_{x x x}+v_{x} \\
v_{t}=(u v)_{x}
\end{array}\right.
$$

Let $\xi=x+c t$, where $c$ is the wave speed. By using the traveling wave transformation $u(x, t)=\varphi(x+c t)=\varphi(\xi), v(x, t)=\psi(x+c t)=\psi(\xi)$, we 
reduce system (1.2) to the following ordinary differential equations:

$$
\left\{\begin{array}{l}
c \varphi^{\prime}-c \varphi^{\prime \prime \prime}+4 \varphi \varphi^{\prime}=3 \varphi^{\prime} \varphi^{\prime \prime}+\varphi \varphi^{\prime \prime \prime}+\psi^{\prime}, \\
c \psi^{\prime}=(\varphi \psi)^{\prime}
\end{array}\right.
$$

Integrating (2.2) once with respect to $\xi$, we have

$$
\left\{\begin{array}{l}
c \varphi-c \varphi^{\prime \prime}=2 \varphi^{2}-\left(\varphi^{\prime}\right)^{2}-\varphi \varphi^{\prime \prime}+\psi+g, \\
c \psi=\varphi \psi+h
\end{array}\right.
$$

where $g, h$ are two integral constants.

From the second expression in system (2.3), we can obtain that

$$
\psi=-\frac{h}{\varphi-c} .
$$

Substituting (2.4) into the first expression in system (2.3) yields

$$
\varphi^{\prime \prime}=\frac{\left(2 \varphi^{2}-c \varphi-\left(\varphi^{\prime}\right)^{2}+g\right)(\varphi-c)-h}{(\varphi-c)^{2}} .
$$

Let $y=\varphi^{\prime}$, then we get the following planar dynamical system:

$$
\left\{\begin{array}{l}
\frac{d \varphi}{d \xi}=y \\
\frac{d y}{d \xi}=\frac{\left(2 \varphi^{2}-c \varphi-y^{2}+g\right)(\varphi-c)-h}{(\varphi-c)^{2}} .
\end{array}\right.
$$

This system has a first integral

$$
H(\varphi, y)=\varphi^{4}-2 c \varphi^{3}+\left(c^{2}+g\right) \varphi^{2}-2(c g+h) \varphi-y^{2}(\varphi-c)^{2}=k,
$$

where $k$ is a constant.

Note that (2.6) has a singular line $\varphi=c$. To avoid the line temporarily, we make transformation $d \xi=(\varphi-c)^{2} d \zeta$. Under this transformation, Eq.(2.6) becomes

$$
\left\{\begin{array}{l}
\frac{d \varphi}{d \zeta}=y(\varphi-c)^{2} \\
\frac{d y}{d \zeta}=\left(2 \varphi^{2}-c \varphi-y^{2}+g\right)(\varphi-c)-h .
\end{array}\right.
$$


System (2.6) and system (2.8) have the same first integral as (2.7). Consequently, system (2.8) has the same topological phase portraits as system (2.6) except for the straight line $\varphi=c$.

For a fixed $k,(2.7)$ determines a set of invariant curves of system (2.8). As $k$ is varied, (2.7) determines different families of orbits of system (2.8) having different dynamical behaviors. Let $M\left(\varphi_{e}, y_{e}\right)$ be the coefficient matrix of the linearized version of system (2.8) at the equilibrium point $\left(\varphi_{e}, y_{e}\right)$, then

$$
M\left(\varphi_{e}, y_{e}\right)=\left(\begin{array}{cr}
2 y_{e}\left(\varphi_{e}-c\right) & \left(\varphi_{e}-c\right)^{2} \\
6 \varphi_{e}^{2}-6 c \varphi_{e}+c^{2}+g-y^{2} & -2 y_{e}\left(\varphi_{e}-c\right)
\end{array}\right)
$$

and at this equilibrium point, we have

$$
\begin{aligned}
& J\left(\varphi_{e}, y_{e}\right)=\operatorname{det} M\left(\varphi_{e}, y_{e}\right)=-3 y_{e}^{2}\left(\varphi_{e}-c\right)^{2}-\left(6 \varphi_{e}^{2}-6 c \varphi_{e}+c^{2}+g\right)\left(\varphi_{e}-c\right)^{2}, \\
& p\left(\varphi_{e}, y_{e}\right)=\operatorname{trace}\left(M\left(\varphi_{e}, y_{e}\right)\right)=0 .
\end{aligned}
$$

By the qualitative theory of differential equations (see [22]), for an equilibrium point of a planar dynamical system, if $J<0$, then this equilibrium point is a saddle point; it is a center point if $J>0$ and $p=0$; if $J=0$ and the Poincaré index of the equilibrium point is 0 , then it is a cusp.

Noting that it is impossible to figure out the equilibrium points of system (2.8) for arbitrary constants $c, g$ and $h$, so here for the sake of convenience, we set $h=\frac{3 c}{32}\left(c^{2}-8 g\right) \neq 0$. By using the first integral value and properties of equilibrium points, we obtain the bifurcation curves as follows:

$$
\begin{aligned}
& g_{1}(c)=-\frac{(16+7 \sqrt{7}) c^{2}}{8}, \\
& g_{2}(c)=\frac{c^{2}}{8}
\end{aligned}
$$




$$
\begin{aligned}
& g_{3}(c)=\frac{3 c^{2}}{8} \\
& g_{4}(c)=\frac{13 c^{2}}{32} .
\end{aligned}
$$

Obviously, the above four curves have no intersection point and $g_{1}(c)<$ $g_{2}(c)<g_{3}(c)<g_{4}(c)$ for arbitrary constant $c \neq 0$.

Using bifurcation method of vector fields (e.g., [20]), we have the following result which describes the locations and properties of the equilibrium points of system (2.8).

Theorem 2.1. For given any constant wave speed $c \neq 0$, let

$$
\varphi_{1 \pm}=\frac{1}{8}\left(5 c \pm \sqrt{13 c^{2}-32 g}\right) \quad \text { for } \quad g \leq g_{4}(c) .
$$

Then we have:

(1) If $g<g_{2}(c)$, then system (2.8) has three equilibrium points $\left(\varphi_{1-}, 0\right)$, $\left(\varphi_{1+}, 0\right)$ and $\left(\frac{c}{4}, 0\right) .\left(\varphi_{1-}, 0\right)$ and $\left(\varphi_{1+}, 0\right)$ are two saddle points, while $\left(\frac{c}{4}, 0\right)$ is a center point. Specially, when $g_{1}(c)<g<g_{2}(c)$, system (2.8) has a homoclinic orbit, which connects with the saddle point $\left(\varphi_{1-}, 0\right)$.

(2) If $g_{2}(c)<g<g_{4}(c)$, then system (2.8) has three equilibrium points $\left(\varphi_{1-}, 0\right),\left(\varphi_{1+}, 0\right)$ and $\left(\frac{c}{4}, 0\right) .\left(\frac{c}{4}, 0\right)$ and $\left(\varphi_{1+}, 0\right)$ are two saddle points, while $\left(\varphi_{1-}, 0\right)$ is a center point. Specially,

(i) When $g_{2}(c)<g<g_{3}(c)$, system (2.8) has a homoclinic orbit, which connects with the saddle point $\left(\frac{c}{4}, 0\right)$.

(ii) When $g=g_{3}(c)$, system (2.8) has two heteroclinic orbits. They connect with the saddle points $\left(\frac{c}{4}, 0\right)$ and $\left(\frac{3 c}{4}, 0\right)$.

(iii) When $g_{3}(c)<g<g_{4}(c)$, system (2.8) has a homoclinic orbit, which connects with the saddle point $\left(\varphi_{1+}, 0\right)$. 
(3) If $g=g_{4}(c)$, then system (2.8) has two equilibrium points $\left(\frac{c}{4}, 0\right)$ and $\left(\frac{5 c}{8}, 0\right)$. $\left(\frac{c}{4}, 0\right)$ is a saddle point, while $\left(\frac{5 c}{8}, 0\right)$ is a cusp.

(4) If $g>g_{4}(c)$, then system (2.8) has only one equilibrium point $\left(\frac{c}{4}, 0\right)$. It is a saddle point.

Without loss of generality, we show the phase portraits of system (2.8) in each region and on the bifurcation curves in Fig 1 for the wave speed $c>0$.

\section{Soliton, kink and antikink solutions of Eqs.(1.2)}

Suppose that $\varphi(\xi)(\xi=x+c t)$ is a traveling wave solution of the first component $u$ in Eqs.(1.2) for $\xi \in(-\infty,+\infty)$, and $\lim _{\xi \rightarrow-\infty} \varphi(\xi)=A, \lim _{\xi \rightarrow+\infty} \varphi(\xi)=$ $B$, where $A$ and $B$ are two constants. If $A=B$, then $\varphi(\xi)$ is called a soliton solution. If $A \neq B$, then $\varphi(\xi)$ is called a kink (or an antikink) solution. Usually, a soliton solution of Eqs.(1.2) corresponds to a homoclinic orbit of system (2.6) and a kink (or an antikink) solution of Eq.(1.1) corresponds to a heteroclinic orbit (or the so-called connecting orbit) of system (2.6) .

In Fig.1(c), (d), (f), the homoclinic orbits of system (2.6) or (2.8) can be expressed respectively as

$$
\begin{aligned}
& y= \pm \frac{\left(\varphi-\varphi_{1-}\right) \sqrt{\varphi^{2}+l_{1-\varphi}+l_{2-}}}{\varphi-c} \text { for } \varphi_{1-} \leq \varphi \leq \varphi_{2-}, \\
& y= \pm \frac{\left(\varphi-\frac{c}{4}\right) \sqrt{\varphi^{2}+l_{1} \varphi+l_{2}}}{\varphi-c} \text { for } \quad \frac{c}{4} \leq \varphi \leq \varphi_{1}, \\
& y= \pm \frac{\left(\varphi-\varphi_{1+}\right) \sqrt{\varphi^{2}+l_{1+\varphi}+l_{2-}}}{\varphi-c} \text { for } \varphi_{2+} \leq \varphi \leq \varphi_{1+},
\end{aligned}
$$

where

$$
l_{1 \pm}=\frac{1}{4}\left(-3 c \pm \sqrt{13 c^{2}-32 g}\right)
$$




$$
\begin{aligned}
& l_{2 \pm}=-\frac{1}{32}\left(-9 c^{2}+16 g \pm c \sqrt{13 c^{2}-32 g}\right), \\
& l_{1}=-\frac{3 c}{2} \\
& l_{2}=-\frac{3}{16} c^{2} g, \\
& \varphi_{1}=\frac{1}{4}\left(3 c-\sqrt{6 c^{2}-16 g}\right), \\
& \varphi_{2 \pm}=\frac{1}{8}\left(3 c \pm\left(-\sqrt{13 c^{2}-32 g}+2 \sqrt{c\left(c+\sqrt{13 c^{2}-32 g}\right)}\right),\right.
\end{aligned}
$$

and $\varphi_{1 \pm}$ is as in (2.16).

Substituting Eq.(3.1) -(3.3) into the first equation of system (2.6), respectively, and integrating along the corresponding homoclinic orbit, we have

$$
\begin{aligned}
& \int_{\varphi}^{\varphi_{2-}} \frac{s-c}{\left(s-\varphi_{1-}\right) \sqrt{s^{2}+l_{1-} s+l_{2-}}} d s=-|\xi|, \\
& \int_{\varphi}^{\varphi_{1}} \frac{s-c}{\left(s-\frac{c}{4}\right) \sqrt{s^{2}+l_{1} s+l_{2}}} d s=-|\xi|, \\
& \int_{\varphi_{2+}}^{\varphi} \frac{s-c}{\left(s-\varphi_{1+}\right) \sqrt{s^{2}+l_{1+} s+l_{2+}}} d s=-|\xi| .
\end{aligned}
$$

It follows from (3.10)-(3.12) that

$$
\begin{aligned}
& \beta_{-}\left(\varphi_{2-}\right)=\beta_{-}(\varphi) \exp (-|\xi|), \\
& \beta\left(\varphi_{1}\right)=\beta(\varphi) \exp (-|\xi|), \\
& \beta_{+}(\varphi)=\beta_{+}\left(\varphi_{2+}\right) \exp (-|\xi|),
\end{aligned}
$$

where

$$
\beta_{ \pm}(\varphi)=\frac{\left(2 \sqrt{\varphi^{2}+l_{1 \pm} \varphi+l_{2 \pm}}+2 \varphi+l_{1 \pm}\right)\left(\varphi-\varphi_{1 \pm}\right)^{\alpha_{1 \pm}}}{\left(2 \sqrt{a_{1 \pm}} \sqrt{\varphi^{2}+l_{1 \pm} \varphi+l_{2 \pm}}+b_{1 \pm} \varphi+l_{3 \pm}\right)^{\alpha_{1 \pm}}}
$$




$$
\begin{aligned}
& \beta(\varphi)=\frac{\left(2 \sqrt{\varphi^{2}+l_{1}+l_{2}}+2 \varphi+l_{1}\right)\left(\varphi-\frac{c}{4}\right)^{\alpha_{1}}}{\left(2 \sqrt{a_{1}} \sqrt{\varphi^{2}+l_{1} \varphi+l_{2}}+b_{1} \varphi+l_{3}\right)^{\alpha_{1}}}, \\
& l_{3 \pm}=\frac{1}{2} c^{2}-2 g \\
& l_{3}=2 g \\
& a_{1 \pm}=\frac{1}{16}\left(13 c^{2}-32 g \pm 3 c \sqrt{13 c^{2}-32 g}\right), \\
& a_{1}=-\frac{1}{8} c^{2}+g, \\
& b_{1 \pm}=\frac{1}{2}\left(c \pm \sqrt{13 c^{2}-32 g}\right), \\
& b_{1}=-c, \\
& \alpha_{1 \pm}=\frac{2 \sqrt{13 c^{2}-32 g-3 c \sqrt{13 c^{2}-32 g}}}{c} \\
& \alpha_{1}=\frac{c}{\sqrt{-2 c^{2}+16 g}},
\end{aligned}
$$

and $l_{1 \pm}, l_{2 \pm}, l_{1}, l_{2}, \varphi_{1 \pm}$ and $\varphi_{2 \pm}$ are as in (3.4), (3.5), (3.6), (3.7), (2.16) and (3.9), respectively.

(3.13)- 3.15 are the implicit expressions for the soliton solutions of the first component $u$ in Eqs.(1.2). From (2.4), (3.13)-(3.15), we can give the parametric expressions for the soliton solutions of the second component $v$ in Eqs.(1.2) as follows:

$$
\left\{\begin{array}{l}
\xi= \pm\left(\ln \beta_{-}(\varphi)-\ln \beta_{-}\left(\varphi_{2-}\right)\right), \quad\left(\varphi_{1-} \leq \varphi \leq \varphi_{2-}\right), \\
\psi=-\frac{3 c\left(c^{2}-8 g\right)}{32(\varphi-c)},
\end{array}\right.
$$




$$
\begin{aligned}
& \left\{\begin{array}{l}
\xi= \pm\left(\ln \beta(\varphi)-\ln \beta_{-}\left(\varphi_{1}\right)\right), \\
\psi=-\frac{3 c\left(c^{2}-8 g\right)}{32(\varphi-c)},
\end{array} \quad\left(\frac{c}{4} \leq \varphi \leq \varphi_{1}\right),\right. \\
& \left\{\begin{array}{l}
\xi= \pm\left(\ln \beta_{+}\left(\varphi_{2+}\right)-\ln \beta_{+}(\varphi)\right), \\
\psi=-\frac{3 c\left(c^{2}-8 g\right)}{32(\varphi-c)},
\end{array} \quad\left(\varphi_{2+} \leq \varphi \leq \varphi_{1+}\right),\right.
\end{aligned}
$$

with $\varphi$ as the parameter.

Now we take a set of data and employ Maple to display the graphs of the above obtained soliton solutions in Figs. 2,4,

In Fig.1(e), the heteroclinic orbits of system (2.6) or (2.8) can be expressed as

$$
y= \pm \frac{\left(\varphi-\frac{c}{4}\right)\left(\varphi-\frac{3 c}{4}\right)}{\varphi-c} \text { for } \quad \frac{c}{4} \leq \varphi \leq \frac{3 c}{4},
$$

Substituting Eq.(3.29) into the first equation of system (2.6), and integrating along the heteroclinic orbits, we have

$$
\left(\varphi_{ \pm}-\frac{c}{4}\right)^{\frac{3}{2}}=\left(\frac{3 c}{4}-\varphi_{ \pm}\right)^{\frac{1}{2}} \exp ( \pm \xi) \quad \text { for } \quad \frac{c}{4} \leq \varphi_{ \pm} \leq \frac{3 c}{4}
$$

where $\varphi_{+}=\varphi_{+}(\xi)$ is a kink solution, and $\varphi_{-}=\varphi_{-}(\xi)$ is an antikink solution.

The corresponding solutions of the second component $v$ in Eqs.(1.2) can be expressed in parametric form as follows.

$$
\left\{\begin{array}{l}
\xi= \pm\left(\frac{3}{2} \ln \left(\varphi_{ \pm}-\frac{c}{4}\right)-\frac{1}{2} \ln \left(\frac{3 c}{4}-\varphi_{ \pm}\right)\right), \\
\psi_{ \pm}=\frac{c^{3}}{16\left(\varphi_{ \pm}-c\right)},
\end{array} \quad\left(\frac{c}{4} \leq \varphi_{ \pm} \leq \frac{3 c}{4}\right),\right.
$$

with $\varphi_{ \pm}$as the parameter. Here $\psi_{+}=\psi_{+}(\xi)$ is an antikink solution, and $\psi_{-}=\psi_{-}(\xi)$ is a kink solution.

Also, we take a set of data and employ Maple to display the graphs of the above obtained kink and antikink solutions in Fig. 5. 
Remark 3.1. (1) The Degasperis-Procesi equation (1.1) does not have kink and antikink solutions because there is no heteroclinic orbit in the corresponding phase portraits (see [23]).

(2) In [24], Vakhnenko and Parkes also suggested a generalized DegasperisProcesi equation. Using direct integration, they obtain its kink and antikink solutions. So the connection between our suggested Eqs.(1.2) and the generalized Degasperis-Procesi equation considered in [24] needs further studying.

(3) The bifurcation method only enables us to analyze the restricted class of solutions of Eqs.(1.2), namely, the one-valued solutions. However the Degasperis-Procesi equation has intriguing solutions such as loop-like solitary wave solutions (see [14]). So whether the 2-component of Degasperis-Procesi equation (1.2) has loop-like solutions needs other mathematical methods.

\section{Conclusion}

In this work, we propose a generalization of the Degasperis-Procesi equation, that is, a 2-component of the Degasperis-Procesi equation (11.2) for the first time. Employing the bifurcation method, we obtain the analytic expressions for smooth soliton, kink and antikink solutions of the 2-component of the Degasperis-Procesi equation (1.2). However, its integrability needs further investigation.

\section{References}

[1] A. M. Wazwaz, Compact and noncompact structures for a variant of KdV equation in higher dimensions, Appl. Math. Comput. 132 (2002) $29-45$. 
[2] Z. Y. Yan, Modified nonlinearly dispersive $m K(m, n, k)$ equations: I. New compacton solutions and solitary pattern solutions, Comput. Phys. Comm. 1 (2003) 25-33.

[3] J. W. Shen, W. Xu, Bifurcations of smooth and non-smooth traveling wave solutions in the generalized Camassa-Holm equation, Chaos, Solitons and Fractals 26 (2005) 1149-1162.

[4] J. H. He, X. H. Wu, Construction of solitary solution and compacton-like solution by variational iteration method, Chaos, Solitons and Fractals 29 (2006) 108-113.

[5] A. Parker, Cusped solitons of the Camassa-Holm equation. I. Cuspon solitary wave and antipeakon limit, Chaos, Solitons and Fractals 34 (2007) 730-739.

[6] Z. R. Liu, Z. R. Ouyang, A note on solitary waves for modified forms of Camassa-Holm and Degasperis-Procesi equations, Phys. Lett. A 366 (2007) 377-381.

[7] A. Biswas, 1-soliton solution of the $K(m, n)$ equation with generalized evolution, Phys. Lett. A 372 (2008) 4601-4602.

[8] Z. J. Qiao, M-Shape peakons, dehisced solitons, cuspons and new 1peak solitons for the Degasperis-Procesi equation, Chaos, Solitons and Fractals 37 (2008) 501-507.

[9] J. B. Zhou, L. X. Tian, Soliton solution of the osmosis $K(2,2)$ equation, Phys. Lett. A 372 (2008) 6232-6234. 
[10] J. B. Li, Y. Zhan, Exact loop solutions, cusp solutions, solitary wave solutions and periodic wave solutions for the special CH-DP equation, Nonlinear Anal.: Real World Appl. 10 (2009) 2502-2507.

[11] J. B. Zhou, L. X. Tian, Solitons, peakons and periodic cusp wave solutions for the Fornberg-Whitham equation, Nonlinear Anal.: Real World Appl. (2009), in press.

[12] A. Degasperis, M. Procesi, Asymptotic integrability, in: A. Degasperis, G. Gaeta (Eds.), Symmetry and Perturbation Theory, World Scientific, Singapore, 1999, pp. 23-27.

[13] A. Degasperis, D. D. Holm, A. N. W. Hone, A new integral equation with peakon solutions, Theo. Math. Phys. 133 (2002) 1463-1474.

[14] V. O. Vakhnenko, E. J. Parkes, Periodic and solitary-wave solutions of the Degasperis-Procesi equation, Chaos Solitons Fractals 20 (2004) 1059-1073.

[15] Y. Matsuno, Multisoliton solutions of the Degasperis-Procesi equation and their peakon limit, Inverse Problems 21 (2005) 1553-1570.

[16] Y. Matsuno, The $N$-soliton solution of the Degasperis-Procesi equation, Inverse Problems 21 (2005) 2085-2101.

[17] Y. Matsuno, Cusp and loop soliton solutions of short-wave models, for the Camassa-Holm and Degasperis-Procesi equations, Phys. Lett. A. 359 (2006) 451-457. 
[18] H. Lundmark, J. Szmigielski, Multi-peakon solutions of the DegasperisProcesi equation, Inverse Problems 19 (2003) 1241-1245.

[19] H. Lundmark, Formation and dynamics of shock waves in the Degasperis-Procesi equation, J. Nonlinear Sci. 17 (2007) 169-198.

[20] J. Lenells, Traveling wave solutions of the Degasperis-Procesi equation, J. Math. Anal. Appl. 306 (2005) 72-82.

[21] C. Chen, M. Tang, A new type of bounded waves for Degasperis-Procesi equation, Chaos, Solitons and Fractals 27 (2006) 698-704.

[22] D. Luo, et al., Bifurcation Theory and Methods of Dynamical Systems, World Scientific Publishing Co., London, 1997.

[23] L. Q. Yu, L. X. Tian, X. D. Wang, The bifurcation and peakon for Degasperis-Procesi equation, Chaos, Solitons and Fractals 30 (2006) 956966.

[24] V. O. Vakhnenko, E. J. Parkes, The solutions of a generalized Degasperis-Procesi equation, Rep. NAS Ukr. 8 (2006) 88-94. 


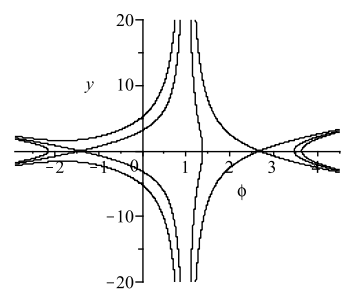

(a)

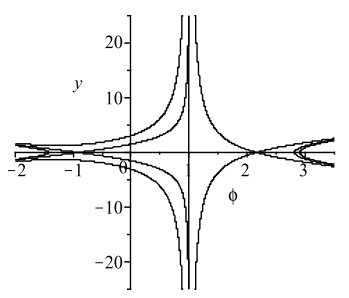

(b)

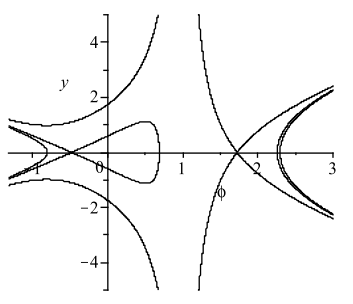

(c)

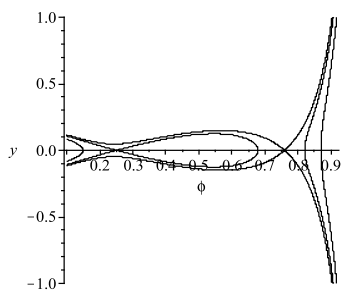

(d)

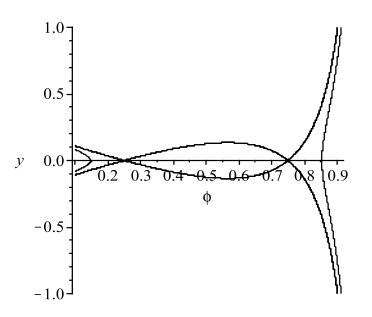

(e)

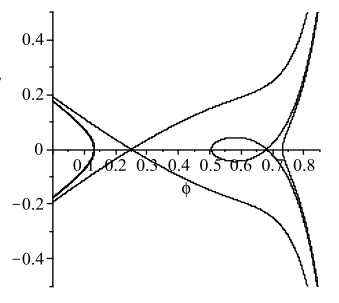

(f)

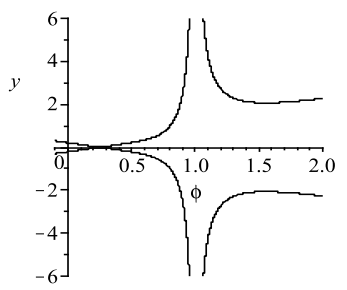

(h)

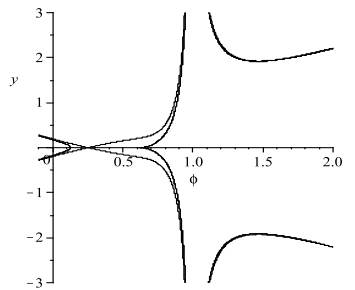

(g)

Figure 1: The phase portraits of system (2.8) for the parameter $c>0$. (a) $g<g_{1}(c)$; (b) $g=g_{1}(c) ;$ (c) $g_{1}(c)<g<g_{2}(c) ;$ (d) $g_{2}(c)<g<g_{3}(c)$; (e) $g=g_{3}(c) ;(\mathrm{f}) g_{3}(c)<g<g_{4}(c) ;(\mathrm{g}) g=g_{4}(c)$; (h) $g>g_{4}(c)$. 


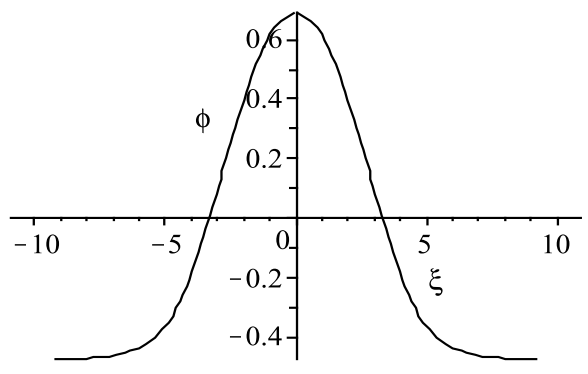

(a)

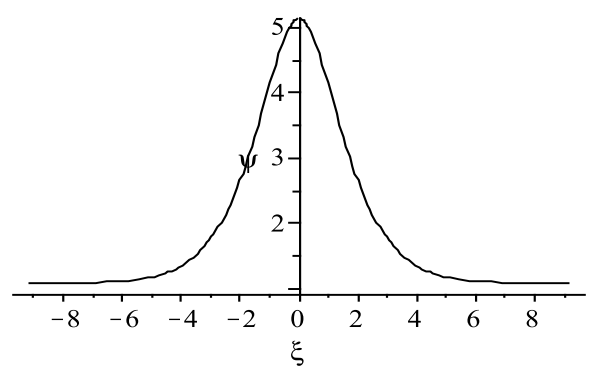

(b)

Figure 2: The soliton of Eqs.(1.2). $(c=1, g=-2, h=1.593750)$

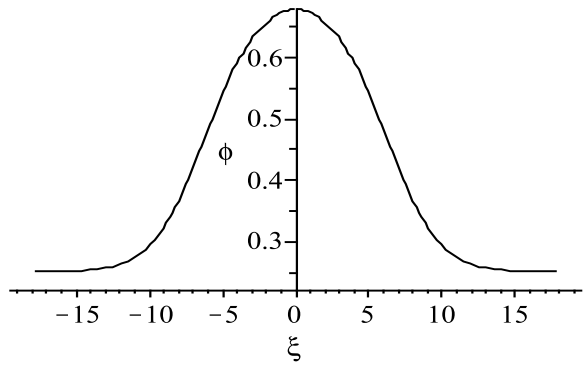

(a)

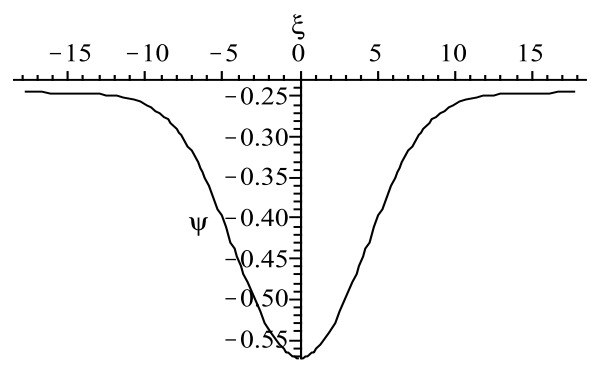

(b)

Figure 3: The soliton of Eqs.(1.2). $(c=1, g=0.37, h=-0.183750)$

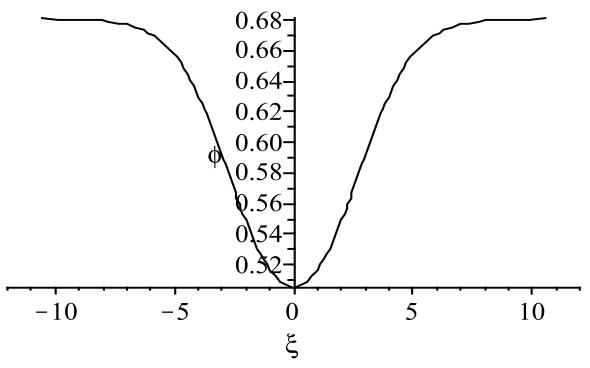

(a)

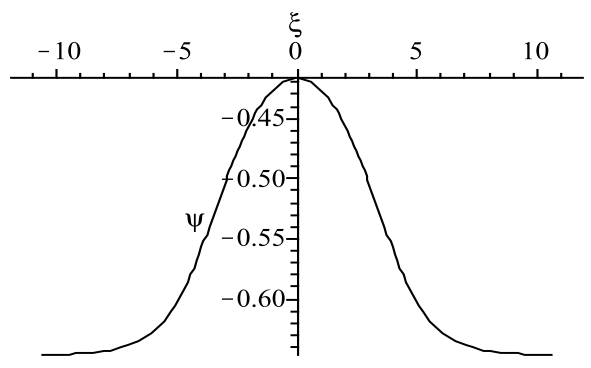

(b)

Figure 4: The soliton of Eqs.(1.2). $(c=1, g=0.4, h=-0.206250)$ 


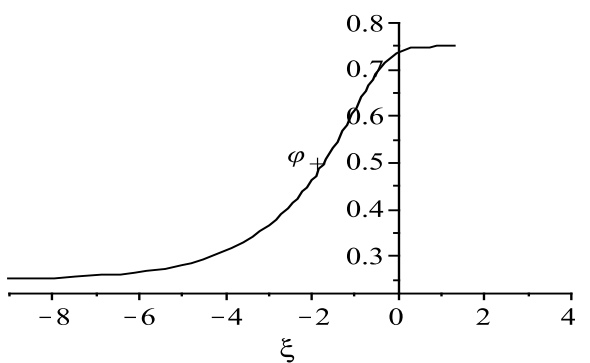

(a)

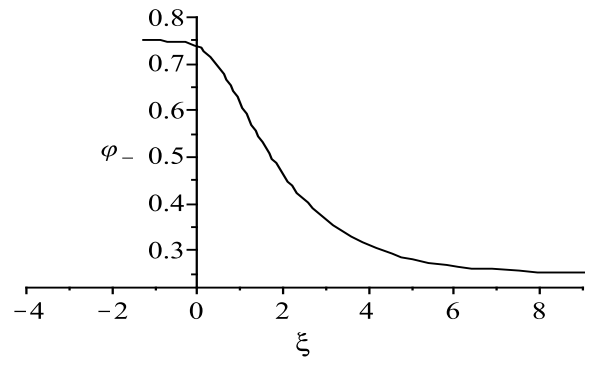

(c)

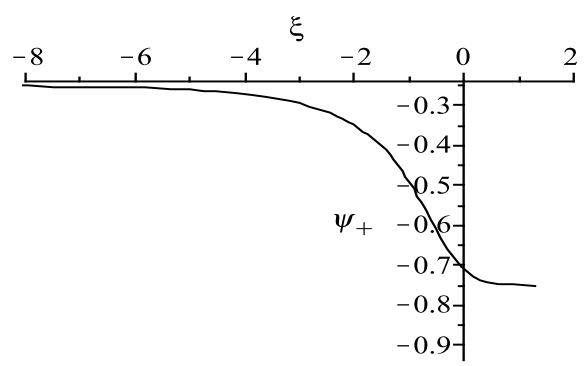

(b)

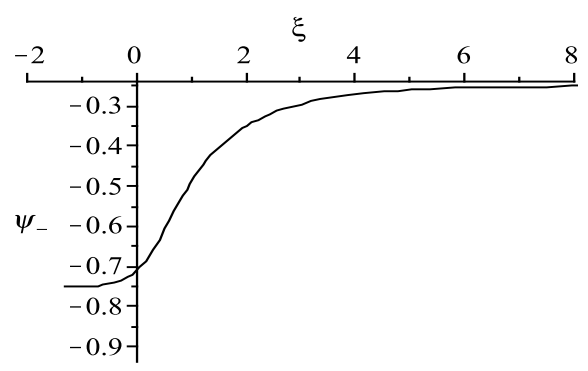

(d)

Figure 5: The kink and antikink wave solutions of Eqs.(1.2). $(c=1, g=$ $0.375, h=0.1875)$ 